\title{
HACIA LA HISPANIDAD A TRAVÉS DE LONDRES. LA INFLUENCIA DE LA ESTANCIA BRITÁNICA DE RAMIRO DE MAEZTU EN LA CREACIÓN DE UN IDEARIO HISPANOAMERICANISTA (1905-1934)
}

\author{
TOWARDS «HISPANIDAD»VIA LONDON. \\ THE INFLUENCE OF RAMIRO DE MAEZTU'S BRITISH PERIOD \\ IN THE CREATION OF A PAN-HISPANIC PROJECT (1905-1934)
}

David Jiménez Torres* Universidad Complutense de Madrid, España

\begin{abstract}
RESUMEN: En su influyente ensayo Defensa de la Hispanidad (1934), Ramiro de Maeztu reivindicó la colonización española de América y argumentó que los rasgos culturales compartidos que resultaban de esa experiencia histórica debían vertebrar una colaboración entre las naciones de habla hispana. Pese a la creencia de que Maeztu desarrolló estas ideas en 1928-30, cuando era embajador en Argentina, sus textos periodísticos muestran que muchas estaban articuladas ya en 1911-12, cuando era corresponsal en Reino Unido. Esto nos anima a revisar el papel del Londres eduardiano en la génesis del ideario hispanoamericanista de Maeztu. Por un lado, le permitió entablar amistad con intelectuales hispanoamericanos como los redactores de la revista Hispania; por el otro, le expuso a los debates que los británicos libraban acerca de su propio Imperio. La Hispanidad aparece así como parte de un movimiento de revisión de la colonización europea y el devenir de los antiguos proyectos coloniales.
\end{abstract}

PALABRAS CLAVE: Hispanidad, Ramiro de Maeztu, Londres, hispanoamericanismo, Reino Unido, España

ABSTRACT: In his influential essay Defensa de la Hispanidad (1934), Ramiro de Maeztu vindicated the Spanish colonization of America and argued that the resulting shared culture of the Spanish-speaking nations should be the basis for political collaboration. Despite the common assumption that Maeztu developed these ideas in 1928-30, when he was Spanish ambassador to Argentina, his journalistic output shows that many of them were developed by 1911-12, when he was working as a foreign correspondent in the United Kingdom. This points to the importance of Edwardian London in the genesis of Maeztu's pan-Hispanism. On the one hand, it allowed him to befriend Latin American intellectuals such as the collaborators of the journal Hispania; on the other, it exposed him to British debates about their own Empire. The "Hispanidad» thus appears as part of a larger revision of the history of European colonization and the future of old colonial projects.

KEYWORDS: Hispanidad, Ramiro de Maeztu, London, hispanoamericanism, United Kingdom, Spain

* Correspondencia a: David Jiménez Torres. Universidad Complutense de Madrid, Departamento de Historia, Teorías y Geografía Políticas, Facultad de Ciencias Políticas y Sociología, Despacho 2610, Campus de Somosaguas, 28223, Pozuelo de Alarcón (Madrid - Spain) - dajimeneztorres@ucm.es - https://orcid.org/0000-0002-8435-0299

Cómo citar: Jiménez Torres, David (2020). «Hacia la Hispanidad a través de Londres. La influencia de la estancia británica de Ramiro de Maeztu en la creación de un ideario hispanoamericanista (1905-1934)»; Historia Contemporánea, 63, 483-509. (https://doi.org/10.1387/hc. 20964).

Recibido: 27 junio, 2019; aceptado: 5 noviembre, 2019.

ISSN 1130-2402 - eISSN 2340-0277 / (C) 2020 UPV/EHU 


\section{Introducción}

La publicación del ensayo Defensa de la Hispanidad en 1934 supone un hito importante en la historia de las propuestas de unidad y colaboración entre España y sus antiguas colonias americanas. Su autor, el periodista y ensayista español Ramiro de Maeztu, escoró la reivindicación de una identidad común y una cooperación política y económica entre las naciones hispanohablantes hacia el terreno del tradicionalismo monárquico español, influyendo así en el devenir de esta corriente de pensamiento. Publicada durante la Segunda República española y con una clara intención de intervenir en los debates de aquel periodo acerca de la religión, la monarquía y la identidad nacional, la obra obtuvo una gran repercusión en España como en algunos países latinoamericanos - especialmente Argentina-. ${ }^{1}$ Tras el asesinato de su autor durante la Guerra civil española, varias de sus tesis fueron incorporadas al ideario del régimen franquista, influyendo sobre su visión de la historia y la identidad nacionales. Además, y como atestiguan sus periódicas reediciones - la más reciente de 2017, con otras en 2011 y 2005 - , ha seguido gozando de una notable salud editorial. De esta forma, las ideas de Maeztu acerca de la Hispanidad suponen uno de los esbozos de proyecto conjunto entre España y sus antiguas colonias más influyentes de la primera mitad del siglo XX.

La génesis de esta obra suele ubicarse en la década de 1920, y en concreto en la experiencia de Maeztu como embajador en Argentina de la dictadura del general Miguel Primo de Rivera. Sin embargo, este trabajo defenderá que Maeztu ya había desarrollado - o adoptado - la mayoría de las tesis que defendería en Defensa de la Hispanidad a comienzos de los años 10, cuando trabajaba como corresponsal en Londres de varios medios españoles e hispanoamericanos. Lejos de encontrarnos ante una cuestión de trascendencia puramente biográfica o anecdótica, también se argumentará que esto tiene repercusiones en cuanto a cómo deberíamos entender el concepto de la Hispanidad desde una perspectiva histórica y contextual. En concreto, que Maeztu desarrollara estas ideas durante sus años en Londres nos anima a reconsiderar el papel desempeñado por Gran Bretaña en la historia de los proyectos culturales y políticos de alcance panhispánico. En primer lugar, por la dimensión de Londres como lugar

\footnotetext{
${ }^{1}$ González Cuevas, 2003, pp. 314-16.
} 
de encuentro de escritores, periodistas y políticos de los distintos países de habla hispana, y por lo tanto como facilitador de dinámicas que daban pie a propuestas de unidad y colaboración. Y, en segundo lugar, por el debate acerca del pasado y el presente del imperialismo europeo en general, y del imperio británico en particular, que se estaba librando en la sociedad británica de finales del XIX y principios del XX - lo que se ha venido a llamar el periodo eduardiano, el que transita desde la muerte de la Reina Victoria en 1901 hasta el estallido de la Primera Guerra Mundial-. Se argumentará que estos debates también suponen un trasfondo significativo a las propuestas hispanoamericanistas que se formularon en las primeras décadas del siglo Xx. Con estos objetivos, primero se hará un resumen de las principales ideas de Defensa de la Hispanidad que permita identificar mejor su génesis. Después se señalarán los motivos por los que cabe sospechar que esta es anterior a lo que se ha venido creyendo, destacando una serie de artículos publicados por Maeztu a finales de 1911 y principios de 1912 que ya contendrían las ideas fundamentales de Defensa de la Hispanidad. Finalmente se explicará el peculiar contexto londinense en el que Maeztu publicó estas obras, incidiendo en su amistad con el grupo de colombianos y peruanos que editaban la revista Hispania, y también los aspectos principales de los debates sobre el imperio que se estaban desarrollando en Reino Unido durante aquellos años. Se concluirá con una reflexión acerca del engarce del hispanoamericanismo de Maeztu en un contexto intelectual europeo más amplio.

\section{La génesis de la Hispanidad}

En líneas generales, Defensa de la Hispanidad supone una reivindicación de las acciones, los valores y el legado del Imperio español en América. Maeztu argumenta que el proyecto imperial español de los siglos XVI y XVII fue esencialmente una acción misionera, que buscó -y consiguió - hacer realidad el sueño católico de extender la posibilidad de alcanzar la salvación a toda la humanidad. El objetivo del colonialismo español no habría sido la explotación de los americanos, sino su incorporación a la comunidad católica y al mundo civilizado. Esta visión, según Maeztu, era compartida por todos los estratos de la sociedad española que intervinieron en el proceso colonizador, de forma que, desde el monarca hasta el último misionero o soldado, los españoles que construyeron el Imperio estaban imbuidos de una idea trascendental de 
su propia misión; una idea marcada por el humanismo y la doctrina católica. Esto explicaría el mestizaje que se había producido en las colonias españolas, en contraste con las británicas; según Maeztu la creencia en una misión trascendente que iba más allá de la mera dominación y explotación había animado a los conquistadores y colonos a mezclarse con los nativos de las tierras que conquistaban, y a tratarlos con la mayor benevolencia posible. Maeztu da el nombre de «Hispanidad» a este proyecto histórico y al tipo de cultura que había fomentado en las comunidades políticas que surgieron tras independizarse de la metrópoli. Y dedica también numerosas páginas a incluir a Portugal y Brasil en este proyecto, proponiendo incluso que la gran obra de la Hispanidad no era el Quijote, sino Os Lusíadas.

Las tesis de Maeztu no implicaban meramente una lectura del pasado; también buscaban actuar como prescripción para el futuro. En su lectura, la plenitud espiritual y geopolítica española de los siglos XVI y XVII se había comenzado a desvanecer cuando España absorbió las ideas materialistas y racionalistas del resto de Europa. La entrada de estas ideas extranjeras durante el siglo XVIII habría provocado, por un lado, la decadencia política, económica y cultural de España, y por otro lado también la independencia de las colonias americanas: estas dejaron de creer en el proyecto común cuando vieron que la metrópoli ya no lo defendía. Sin embargo, tampoco las nuevas repúblicas habían alcanzado una verdadera plenitud tras su emancipación. El resultado era la postración geopolítica en la que se encontraban las naciones latinoamericanas a comienzos del siglo XX, amenazadas tanto por el imperialismo estadounidense como por el expansionismo soviético, y testigos del colapso de sus otros referentes europeos (como Francia o Gran Bretaña). Por todo esto, Maeztu argumentaba que ni España ni sus antiguas colonias recuperarían la plenitud perdida a menos que volviesen los ojos sobre su antigua y compartida identidad hispánica, y dejaran que esta influyese en su cultura política y su acción de gobierno. Además, y con el ojo puesto en la España republicana, Maeztu reivindicaba el sistema monárquico como uno de los valores constitutivos de la Hispanidad; y no una monarquía constitucional al modelo decimonónico, sino una monarquía ejecutiva y católica, imbuida en los valores de jerarquía y de servicio que habrían dado sentido a la institución en el pasado.

A la hora de reivindicar una mayor unidad de acción entre España y sus antiguas colonias americanas, Maeztu negó explícita y repetidamente que estuviese proponiendo una actualización del imperialismo: 
«A mí no me gusta la palabra Imperio, que se ha echado a volar en estos años. No tengo el menor interés en que empleados de Madrid vuelvan a recaudar tributos en América. Lo que digo es que los pueblos criollos están empeñados en una lucha de vida o muerte con el bolchevismo, de una parte, y con el imperialismo económico extranjero, de la otra, y que si han de salir victoriosos, han de volver por los principios comunes de la Hispanidad». ${ }^{2}$

Según Maeztu, la afinidad cultural y la coordinación intergubernamental no requerían la unidad política, sino todo lo contrario. En su opinión, la decadencia del Imperio español había comenzado en el momento en el que dejó de creer en una misión trascendente y se centró exclusivamente en ser un organismo político y económico. A este respecto, escribió que era «esencial subrayar la pluralidad que el concepto de Hispanidad lleva implícito, porque nada haría tanto daño a nuestra idea del Nuevo Mundo como darle un tinte de imperialismo». Y también argumentó que la resurrección de la Hispanidad solo se podría llevar a cabo si las repúblicas latinoamericanas «se nacionaliza[n] aún más de lo que están. Los argentinos han de ser más argentinos; los chilenos, más chilenos; los cubanos, más cubanos». ${ }^{3}$

Pese a esto, la Hispanidad de Maeztu ha sido objeto de varias lecturas críticas que se centran en su relación funcional con un proyecto o una mentalidad imperial y eurocéntrica. Sepúlveda ha argumentado que «la Hispanidad es, en realidad, un intento de retrotraer el papel de España a una etapa donde existía una situación privilegiada y de dominio». ${ }^{4}$ Centrando la discusión en Maeztu, José Luis Abellán escribió sobre la «voluntad imperial» de Defensa de la Hispanidad, y Alistair Hennessy afirmó que el proyecto de Maeztu representaba un «imperialismo sucedáneo». ${ }^{5}$ Estas lecturas destacan que Maeztu, al fin y al cabo, exalta un proyecto imperial, el de la España de los siglos XVI y XVII; y que no solo lo alaba por los valores que lo habrían animado, sino también por lo exitoso que fue aquel proyecto, al haber conseguido «asimilarse a su propia civilización cuantas razas de color sometió». ${ }^{6}$ Así, el mestizaje que Maeztu reivindica no es tanto un intercambio en pie de igualdad sino una absorción

\footnotetext{
2 Maeztu, 2005, p. 223.

3 Ibid.

4 Sepúlveda, 2005 p. 162.

5 Abellán, 1973, p. 157; Hennessy, 2000, p. 106.

6 Maeztu, 2005, p. 83.
} 
coercitiva; no puede ser de otra manera cuando su catolicismo y su rechazo al concepto del «buen salvaje» le llevan a descartar la idea de que los pueblos indígenas pudieran haber estado en mejor situación antes de la colonización española que después, o que podrían haber aportado algo a la cultura o la civilización de la metrópoli. Por otro lado, la necesaria nacionalización de los argentinos, chilenos y cubanos que defiende está acotada en su desarrollo, ya que «no 1[a] conseguirán si no son al mismo tiempo más hispánicos, porque la Argentina y Chile y Cuba son sus tierras, pero la Hispanidad es su común espíritu». ${ }^{7}$ Las lecturas críticas de la Hispanidad también amplían el foco hacia el contexto histórico, señalando - de nuevo desde una perspectiva funcional que trasciende las declaraciones Maeztu sobre sus intenciones - que la Hispanidad sería el producto de un trauma posimperial tras la pérdida de las últimas colonias americanas, un empeño por sustituir la función desempeñada en el imaginario colectivo por un imperio político transatlántico - el que había llegado a su fin en 1898 - por la de un imperio cultural. ${ }^{8}$

Lo que nos interesa en este trabajo, sin embargo, no es tanto en qué punto del espectro imperialismo/anti-imperialismo - cuyas peculiares coordenadas durante la época se comentarán más adelante - ubicar las ideas de Maeztu, sino trazar la génesis de las mismas. Como se señaló al comienzo, la interpretación dominante es que Maeztu habría desarrollado su concepto de la Hispanidad entre 1928 y 1930, y en el contexto de su etapa como embajador en Argentina de la dictadura de Primo de Rivera. ${ }^{9}$ Esta interpretación se apoya en varios hechos. En primer lugar, fue allí donde Maeztu conoció a Zacarías de Vizcarra, el clérigo español a quien él mismo atribuiría haber acuñado el término «Hispanidad». ${ }^{10}$ También fue en Argentina donde Maeztu entró en contacto con el grupo intelectual y político organizado alrededor de la revista La Nueva República, que reivindicaba el catolicismo como principal componente de la identidad na-

7 Maeztu, 2005, p. 223.

${ }^{8}$ Marcilhacy 2016, pp. 146-151.

9 Ejemplos de esta interpretación, en Vegas Latapie, 1983, p. 264; Landeira, 1978, p. 42; Abellán, 1973, p. 152; Mainer, 2004, p. 19. Marcilhacy (2010, p. 269) ha llamado la atención sobre un artículo de Maeztu de 1927 en el que ya avanzaba sus ideas sobre la Hispanidad: «Plus Ultra. El mito de la Raza», El Sol 9 de enero de 1927. En esta versión, el hispanoamericanismo de Maeztu sería inmediatamente anterior a su designación como embajador, y posiblemente incluso la explicaría.

${ }_{10}$ González Cuevas, 2003, p. 244. Este autor nos recuerda que, en realidad, Unamuno ya había utilizado el término unos años antes. 
cional y miraba positivamente a la tradición cultural hispana. Además, parte de su labor como embajador consistió en pronunciar una serie de conferencias que reivindicaban la fraternidad entre España y sus antiguas colonias, conferencias que terminaron convertidas en varios capítulos de Defensa de la Hispanidad. Esto también encaja con las interpretaciones que acabamos de ver sobre la función y el significado de la Hispanidad: al fin y al cabo, la dictadura de Primo de Rivera había convertido en prioritaria la relación política y económica con las repúblicas hispanoamericanas, y había incorporado a su discurso muchos de los argumentos del hispanoamericanismo, bien que adaptándolos a un proyecto conservador, nacionalista y monárquico. ${ }^{11}$ Desde este punto de vista, la Hispanidad habría germinado como una herramienta diplomática, una suerte de «poder blando» avant-la-lettre puesto en marcha por la derecha monárquica española de los años veinte con el objetivo de reforzar alianzas internacionales y moldear una cultura favorable a sus intereses políticos. ${ }^{12}$

Sin embargo, hay razones para afirmar que el hispanoamericanismo de Maeztu se desarrolló muchos años antes de ser nombrado embajador en Argentina. Cuanto menos, podemos encontrar su primera manifestación en cuatro artículos que publicó entre finales de 1911 y comienzos de 1912. ${ }^{13}$ Maeztu llevaba por entonces siete años viviendo en Londres, adonde había llegado en 1905 como corresponsal de prensa. Si bien los temas de los que solía escribir eran los relacionados con la cultura y la política británicas, o con las distintas recetas para la modernización de España, decidió aparcar esto para dedicar algunas reflexiones al futuro de Hispanoamérica. En estos artículos, Maeztu se mostraba alarmado ante la posibilidad de que Estados Unidos expandiera su influencia política y cultural sobre el resto de repúblicas americanas. En su opinión, esto supondría nada menos que «un desastre para la humanidad», dado que la influencia de los estadounidenses sobre el resto del continente solo podía ser materialista, y por tanto contraria al sustrato idealista de la cultura hispanoamericana. Juntando a EE UU y a Reino Unido como dos mani-

\footnotetext{
11 Niño Rodríguez, 1993; González Calleja y Limón Nevado, 1988.

12 Ocio, 2014, pp. 157-178. La idea del «poder blando» está desarrollada en Nye, 2004.

13 Se trata de: Maeztu, R. de., «Americanismo», Nuevo Mundo, 14 de septiembre de 1911; «Los "indianos"», Nuevo Mundo, 30 de noviembre de 1911; «El ideal de la fraternidad», Nuevo Mundo, 18 de enero de 1912; «Apología de los “antis”», Nuevo Mundo, 25 de enero de 1912.
} 
festaciones de una misma cultura, Maeztu señalaba que los «pueblos anglosajones» tenían una concepción racista del dominio sobre pueblos extranjeros, lo que les llevaba a afrontaban la colonización como un mero cálculo de ganancias materiales, en vez de como una misión espiritual. Por ello, y de nuevo según Maeztu, los anglosajones no sentían la necesidad de mezclarse con los nativos de sus colonias, ni de permitirles participar en la gestión de las mismas. Esto, a su vez, demostraba por contraste

«la inmensa superioridad de la colonización española en América sobre la colonización anglosajona. En Colombia, en Venezuela, en CentroAmérica, en Méjico, en el Brasil [...] se ha llegado a realizar la fusión de las razas humanas y se ha resuelto hace cuatro siglos el problema que hace bambolearse la civilización sajona».

Maeztu concluía de todo esto que «esta es la obra gloriosa e inmortal del genio de España. España ha hecho posible en América la unidad material del género humano».

Partiendo, por tanto, de la idea de que el mundo hispanohablante el resultado de una misión transcendente y unificadora, el Maeztu de 1911-12 lamentaba que las relaciones afectivas e intelectuales entre las naciones hispanas fuesen, en su opinión, demasiado débiles. Y resultaba urgente revertir esta situación, puesto que sin una mayor cooperación ni los españoles ni los latinoamericanos podrían «cumplir con su deber supremo de dar su cuño especial a la cultura humana, enriqueciéndola con una cultura hispánica que sea superior, en su momento, a la de otras razas». El eje de este proyecto sería, por tanto, la cultura; pero Maeztu abría la puerta también a las consecuencias políticas y diplomáticas. Es más, señalaba que reforzar los lazos entre las naciones hispánicas de ambos lados del Atlántico lograría «evitar que los norteamericanos extiendan su dominio en Hispano-América, y los franceses, ingleses y alemanes su dominio en España». Así, Maeztu sugería que la unidad cultural entre España y sus antiguas colonias era la condición previa y necesaria para una colaboración política, económica y militar en clave defensiva ante naciones más ricas y poderosas. Este énfasis en garantizar la autonomía de cada uno de los países que integraran este proyecto excluía, también, cualquier deseo de restablecer el antiguo Imperio español:

«No se trata, naturalmente, de reconstituir imperios coloniales, ni de colocar las más débiles de las naciones hispano-parlantes bajo la tu- 
tela de las más fuertes. Todo movimiento de fraternidad, de solidaridad, ha de tener por base la confirmación y aún el fortalecimiento de cada una de las nacionalidades que hablan nuestro idioma».

Como podemos ver, estos artículos ya contienen muchos de los argumentos que Maeztu plasmaría años después en Defensa de la Hispanidad. Está presente en ellos, por ejemplo, la reivindicación de la experiencia colonial española como una labor misionera que se habría cimentado en un compromiso con la igualdad ontológica entre los seres humanos - lo que, a su vez, habría sido posible gracias a una base cultural y teológica únicamente católica-. También encontramos la idea de que España y sus antiguas colonias comparten una esencia y una identidad basadas en el antimaterialismo, y que esto les enfrenta necesariamente a otras culturas. Encontramos, además, el uso ambiguo del concepto raza, con connotaciones culturales e historicistas más que biologizantes, y que se convertiría en un eje importante del nacionalismo conservador en su relación con las coordenadas intelectuales del hispanoamericanismo. ${ }^{14} \mathrm{Y}$, por último, encontramos la afirmación de que este proyecto no implicaría establecer (ni restablecer) el dominio político de cualquiera de las partes sobre las demás. Por otra parte, no hay rastro en estos textos de un aspecto importante de Defensa de la Hispani$d a d$, que es la defensa de la monarquía como forma de gobierno; y también es notable la diferencia de énfasis en el catolicismo como fuente de los valores hispánicos: si en los años 10 Maeztu trata el asunto con un vocabulario orteguiano y neokantiano, para finales de los años 20 el vocabulario se ha vuelto mucho más menendezpelayista. Sin embargo, y en rasgos generales, estos artículos dan fe de que buena parte del ideario hispanoamericanista y el interés de Maeztu por un proyecto conjunto entre España y sus antiguas colonias ya estaba presente a mediados de su etapa londinense.

Esto nos anima a reconstruir la génesis de su concepto de Hispanidad. Por un lado, podemos apuntar algunos factores biográficos que ayudarían a explicar el interés de Maeztu por la relación pasada, presente y futura entre España y sus antiguas colonias. El vínculo con Cuba había marcado fuertemente su historia familiar y personal: los Maeztu habían sido propietarios de varios negocios en aquella isla cuando esta todavía

14 Marcilhacy, 2010. 
se encontraba bajo dominio español. Las dificultades que aquellos negocios sufrieron la adolescencia del joven Ramiro le hizo acompañar a su padres a Cuba para tratar de enderezar la situación. Allí, Maeztu había llegado a conocer bien la sociedad y la cultura de aquella isla antes de que su padre - quien moriría escasos meses después - le enviara de vuelta a España. ${ }^{15}$ Estas experiencias habían influido, por su parte, en el análisis que Maeztu realizó de las guerras coloniales durante sus primeros años como articulista, en piezas como las recogidas en la sección «De las guerras» de su primer ensayo Hacia otra España (1899), y en las que desarrollaba una visión más crítica y realista de lo que era habitual en la prensa del momento. ${ }^{16}$ Además, una de las cabeceras para las cuales Maeztu realizaba su labor como corresponsal en Londres era el diario argentino La Prensa, al que permanecería vinculado hasta su regreso a España en 1919. ${ }^{17}$

Además de estos factores biográficos podemos apuntar a otros de cariz contextual. Los cuatro artículos de Maeztu que se han citado se publicaron en el contexto de los centenarios de la independencia de las repúblicas hispanoamericanas (1910-1924, si bien algunos de los más sonados - Argentina, México, Chile y Venezuela - se concentraron en los dos primeros años). Lejos de tratarse de celebraciones puramente nacionales, tuvieron un amplio eco transatlántico y contaron con presencia institucional española. ${ }^{18}$ También era la época inmediatamente posterior al viaje de Rafael Altamira a Hispanoamérica, donde pronunció unas 300 conferencias en siete países, y a su igualmente triunfal regreso a España, donde se le brindó un gran recibimiento institucional y popular. Este contexto reforzó los discursos y postulados hispanoamericanistas que se llevaban formulando a ambos lados del Atlántico desde mediados del siglo XIX, y que reivindicaban en líneas generales una mayor cercanía y cooperación entre las repúblicas hispanoamericanas y España, de acuerdo con su historia e intereses compartidos. ${ }^{19}$

En su vertiente española, este discurso exhibió una notable transversalidad ideológica; su principal reclamación era que la antigua metrópoli ejerciera un papel de influencia preferente sobre sus antiguas colonias, si

\footnotetext{
15 González Cuevas, 2003, pp. 38-43.

16 Maeztu, 2007, pp. 91-144.

17 Castro Montero, 2012, pp. 19-50.

18 Moreno Luzón, 2010.

19 Pike, 1971; Lombardero Álvarez, 1999; Sepúlveda, 2005.
} 
bien se solía enfatizar que dicha influencia debía ejercerse desde un plano de igualdad política. También se reivindicaba la labor colonizadora de España en Hispanoamérica y su presunta vocación de mestizaje, en contraposición con la actitud desarrollada por los anglosajones en su colonización de y expansión por Norteamérica. ${ }^{20}$ Una parte importante de este discurso era la refutación explícita de la leyenda negra, proyecto que encontró otro gran impulso en estos años con la publicación del ensayo de Julián Juderías La leyenda negra (1914). En cualquier caso, de este discurso no participaban solamente gobiernos nacionales y/o municipales, sino también un nutrido tejido civil y asociativo compuesto por asociaciones de inmigrantes, cámaras de comercio, ateneos, etc. Todo esto alimentaba, además, las redes de contactos entre españoles y americanos, en las que también tuvieron especial importancia los viajes a un lado y otro del Atlántico de escritores e intelectuales.

Muchas de las ideas que Maeztu recoge en los artículos de 1911-12 tenían, por tanto, una larga trayectoria en la cultura española, e incluso podemos decir que se encontraban en el aire cultural de aquellos años; por no hablar de que la biografía de Maeztu le habría hecho especialmente receptivo a ellas. Sin embargo, también existen motivos para relativizar el poder explicativo de estas influencias, al menos en lo que a Maeztu se refiere. Por un lado, los artículos acerca de las guerras coloniales recogidos en Hacia otra España muestran que el joven Maeztu trajo de Cuba una serie de tesis sobre el conflicto colonial pero no una idea articulada acerca de las futuras relaciones entre España y sus antiguas colonias; resulta evidente que eso lo desarrolló más tarde. En lo que se refiere a su interpretación histórica de la colonización española durante los años anteriores a su etapa londinense, Maeztu no se desmarca del relato republicano clásico según el cual el imperio habría sido una sangría de población y recursos para España. ${ }^{21}$ Por otro lado, Maeztu se mostró muy crítico en sus artículos de 1911-12 precisamente con el tipo de discursos y de conmemoraciones realizados durante los centenarios: «nada más absurdo que celebrar banquetes en los que se da por supuesta

20 Ver, por ejemplo, Rodríguez, «El Centenario de la Independencia americana», Unión Ibero-Americana, julio de 1908; y Salaverría, «Altamira en América», ABC, 6 de abril de 1910. Mi agradecimiento a Javier Moreno Luzón por llamar mi atención sobre estos textos.

${ }^{21}$ Por ejemplo, los artículos «Sobre el discurso de Lord Salisbury», «La vara de medir» $\mathrm{y}$ «Responsabilidades», recogidos en Maeztu, 2007, pp. 125-27, 135-36 y 139-41. 
la fraternidad hispano-americana y se busca la manera de «estrechar lazos»... que no existen. Todo es «retórica de espuma de champaña». ${ }^{22}$ Y, lo que es más importante, no debemos perder de vista que en el momento de los centenarios Maeztu llevaba varios años asentado en la capital británica, y con una implicación cada vez mayor en su vida cultural e intelectual. Había trabado amistad con el editor A. R. Orage y el poeta y filósofo T. E. Hulme, quienes se convertirían en sus interlocutores más asiduos; y a través de ellos conocería a personajes fundamentales de la vanguardia británica como Wyndham Lewis, Jacob Epstein o Ezra Pound. En 1913 publicó su primer artículo en The New Age, revista editada por Orage que se encontraba entre las publicaciones de referencia de la izquierda británica del momento, y de la que Maeztu se convertiría en colaborador asiduo. Además, en el transcurso de sus primeros años de estancia londinense Maeztu se había acercado a varios movimientos político-culturales que no tenían un equivalente claro en el contexto español: el socialismo fabiano, el new liberalism, el socialismo gremial, el ideal de la «eficiencia nacional», el distributismo de Hilaire Belloc y G. K. Chesterton e incluso el modernismo católico. ${ }^{23}$ Por contraste, durante estos años Maeztu no parecía sentir mucho interés por los debates sobre el «ser de España» que animaban la producción intelectual de varios de sus compañeros de generación. ${ }^{24}$ Así, no sorprende que Maeztu confesara en una carta a Ortega su impresión de encontrarse muy desconectado de las coordenadas de debate intelectual de la España de aquellos años: "prefiero poner mi esfuerzo al servicio de la causa del socialismo gremial - causa que es, por ahora, genuinamente inglesa- que no batallar en España sin ninguna clase de rumbo. Y lo que me molesta, sobre todo, es el exotismo, única relación que parece existir entre mi espí-

22 «El ideal de la fraternidad», Nuevo Mundo, 18 de enero de 1912.

23 Para estos autores, publicaciones y movimientos, ver Martin, 1967; Ferguson, 2002; Lothian, 2009; Corrin, 1981 y 2002; Freeden, 1978; Searle, 1971. Un análisis detallado de su influencia en la evolución ideológica de Maeztu, en Jiménez Torres, 2016 a.

${ }^{24}$ Recordemos que, durante los años que Maeztu pasó en Londres, Azorín publicó La ruta de don Quijote (1905), Castilla (1912) y Clásicos y modernos (1913), Antonio Machado publicó Campos de Castilla (1912), Unamuno publicó Por tierras de Portugal y España (1911) y Ortega publicó Meditaciones del Quijote (1914). Por contraste, la obra que culmina la etapa británica de Maeztu, Authority Liberty and Function in the Light of the War (1916; traducida al español como La crisis del humanismo, 1919) no muestra ningún interés ni por el concepto de nación en general ni por el de la española en particular. 
ritu y su ambiente español». ${ }^{25}$ Por todo esto, conviene examinar también el contexto londinense del momento y comprobar de qué forma pudo haber influido en el interés de Maeztu por el hispanoamericanismo y la forma que iban tomando sus ideas acerca de la relación pasada y futura de España con sus antiguas colonias.

\section{Londres: lugar de encuentro y cuna de Hispania}

Pese a lo que pueda parecer, Londres era, a comienzos del siglo Xx, un buen sitio para pensar la Hispanidad. En primer lugar, porque la capital británica era un importante lugar de encuentro de escritores y pensadores del mundo hispánico. Ya los liberales españoles que se debieron exiliar en Londres durante el reinado de Fernando VII habían entrado en contacto en aquella ciudad con figuras de las nuevas repúblicas latinoamericanas, como Vicente Rocafuerte, Manuel Lorenzo Vidaurre, José Antonio Miralla o José Miguel Ramos Arizpe; esto había dado pie a algunos de los primeros escritos que reivindicaban una identidad compartida entre las nuevas repúblicas latinoamericanas y la antigua metrópolis. ${ }^{26}$ Varias décadas después, Maeztu se encontró dinámicas similares. De hecho, la importancia y frecuencia de su contacto en Londres con intelectuales hispanoamericanos a veces queda eclipsada en los estudios sobre su etapa inglesa, atrayendo mayor atención su contacto con autores y movimientos intelectuales de Inglaterra y de Alemania. ${ }^{27}$ Sin embargo, el economista español Luis Olariaga, quien también vivió en Londres durante aquellos años y tuvo trato frecuente con Maeztu, señaló que durante toda su etapa inglesa el corresponsal «recibía, a menudo, visitas de políticos de categoría y de diplomáticos de Hispanoamérica, a los que introducía en los centros oficiales que les interesaban». ${ }^{28}$ Esto no es sorprendente si tenemos en cuenta que Maeztu era muy conocido en Hispanoámerica debido a sus colaboraciones en La Prensa de Buenos Aires, uno de los periódicos más influyentes y con mayor distribución de la época; el mismo Olariaga señaló que, en su opinión, Maeztu reservaba sus mejores artículos para

\footnotetext{
25 Epistolario Maeztu/Ortega, FOGM, carta de 21 de abril de 1915.

26 Bermejo Marín, 2017.

27 Por ejemplo, en Fraga 1974 y 1976.

28 Olariaga, 1974, pp. 55. Para más testimonios de personas que le trataron durante estos años, ver Pla, 1952, y Madariaga, 1974.
} 
aquella cabecera. ${ }^{29}$ También debido a esta colaboración, Maeztu realizaría al menos una de sus visitas al frente como corresponsal de guerra durante la Primera Guerra Mundial junto a un grupo de periodistas latinoamericanos. ${ }^{30}$

Esta colaboración también fomentaba el interés del propio Maeztu por las realidades de las repúblicas latinoamericanas. El escritor y político argentino Ricardo Rojas, quien trabó amistad con Maeztu durante una estancia que realizó en Londres en 1908, escribió que «él era entonces corresponsal de La Prensa de Buenos Aires y esto lo acercaba a todo lo argentino». ${ }^{31}$ Pero su interés no se limitaba a este país. También trató en Londres al escritor y diplomático boliviano Alcides Arguedas, quien le pidió que escribiera el prólogo de su ensayo Pueblo enfermo (1909), obra que aplicaba algunos de los esquemas del regeneracionismo costista a la situación de Bolivia. En dicho prólogo, Maeztu indica que considera homologables las preocupaciones de las élites reformistas de las naciones latinoamericanas a las de las españolas. ${ }^{32}$ Dirigiéndose a Arguedas, señala que «usted ha hecho por su país, con este libro, lo que unos cuantos españoles hicimos por el nuestro hace diez años, a raíz de haberse perdido las colonias. Nos apartamos espiritualmente de él para verlo mejor desde fuera»; e indicaba que los reformistas españoles y latinoamericanos eran miembros de una única y gran generación: «al analizar nuestras dudas nos damos cuenta de que son las dudas de nuestra generación, [...] la que ha de realizar la obra reformista». ${ }^{33}$ Maeztu también mostraba en este breve texto su opinión de que las sociedades hispanohablantes compartían algunos patrones de comportamiento, sobre todo en lo que se refería a la actitud ante los presuntos redentores: «los pueblos de habla española no somos tanto los de Don Quijote como los que clavamos a Don Quijote en la picota del ridículo». ${ }^{34}$ Por otro lado, el propio Rojas dio fe de que fue la combinación de su propio interés por España y el de Maeztu por Argentina lo que hizo que ambos llegaran a «la más completa intimidad intelectual». 35

\footnotetext{
29 Ibid.

30 Maeztu, 1916.

31 Rojas, 1938, p. 251.

32 Sierra Gutiérrez, 1984, p. 64.

33 Arguedas, 1909, p. 8, 14.

34 Ibid., p. 9.

35 Rojas, op. cit.
} 
Otra amistad relevante fue la que Maeztu entabló durante sus primeros años en Londres con el escritor y diplomático peruano Francisco García Calderón. ${ }^{36}$ Este era un seguidor de las ideas propuestas por José Enrique Rodó en el celebérrimo ensayo Ariel, y por tanto un defensor de una identidad compartida de las repúblicas latinoamericanas, centrada en el idealismo y el anti materialismo. Su ensayo La creación de un continente (1912) criticaba la labor histórica de los nacionalismos de cada una de las repúblicas hispanoamericanas -incluyendo sus manifestaciones en el contexto de los festejos de los centenarios de independencia-, postulando que aquellas debían unirse en base a su pasado común y a sus intereses compartidos. La herencia de la cultura española, y las amenazas geopolíticas que se cernían sobre las repúblicas hispanoamericanas, debían ser el nexo para la cooperación política, económica y cultural. ${ }^{37}$

En una obra anterior, Profesores de idealismo (1909), García Calderón dedicó un capítulo a Maeztu en el que daba fe de la amistad entre los dos, sustentada en numerosas charlas en el Devonshire Club, que ambos frecuentaban. ${ }^{38}$ García Calderón se muestra muy elogioso con Maeztu, alabando tanto su personalidad como su pensamiento, y recuenta una larga conversación entre ambos acerca de La Tempestad y las reservas de Maeztu acerca de la lectura alegórica de aquella obra que Rodó había hecho en clave latinoamericana - y latinoamericanista - . Es difícil saber si Maeztu ya se había interesado por el arielismo antes de conocer a García Calderón o si esta amistad le había animado a conocer y adoptar algunos de sus postulados, como la visión de Hispanoamérica como una esfera cultural unificada $-\mathrm{y}$ no un grupo heterogéneo de naciones - , o la identificación de esa cultura compartida con el idealismo filosófico. Pero García Calderón da fe de la atención que su amigo daba a estos temas: «Este es el pensamiento de Maeztu. Cree que hay que dar ideas, en clara forma, en pródiga abundancia, para que de ellas viva la mentalidad común de la raza española, allende y aquende el Atlántico. Y por eso espera y por eso cree en el porvenir hispano». ${ }^{39}$

García Calderón formaba parte, además, de un grupo de intelectuales hispanoamericanos vinculados a Hispania, revista cultural que se editaba

\footnotetext{
36 González Cuevas, 2003, p. 117.

37 Bruno, 2018, pp. 57-77.

38 García Calderón, 1909, p. 111.

39 Ibid., p. 116.
} 
en Londres durante estos años. ${ }^{40}$ Maeztu y él se habían conocido precisamente en una charla impartida por el fundador de la revista, el exiliado liberal colombiano Santiago Pérez Triana; y entre los colaboradores de la misma destacaban los también colombianos Baldomero Sanín Cano y Enrique Pérez Lleras. El objetivo de aquella publicación, también influida fuertemente por el arielismo, era fomentar los lazos y el conocimiento mutuo entre los países hispanoamericanos. Precisamente porque Pérez Triana y sus colaboradores consideraban que España debía formar parte de esa esfera de cooperación, la revista se esforzó por implicar en su proyecto a escritores y periodistas españoles y contó con colaboraciones de figuras como Unamuno, Altamira, Pérez de Ayala, Azorín y Luis Araquistain. También contó con un mediador cultural excepcional entre Reino Unido, España y las repúblicas latinoamericanas como era R. B. Cunninghame Graham.$^{41}$ La actividad del grupo de Hispania rebasaba a la propia publicación: Pérez Triana organizaba reuniones formales e informales en las que intelectuales de distintos países latinoamericanos coincidían y debatían con comparsas españoles, británicos y franceses. No sorprende, por tanto, que Maeztu se refiriese a Hispania en sus artículos de 1911-12 como un ejemplo del tipo de proyecto cultural que él mismo estaba proponiendo, y como una demostración de que este «no se piensa tan solo entre los españoles de la península, [sino] que también es objeto de meditaciones por parte de los hispanoamericanos». Que autores del otro lado del Atlántico defendieran sus mismos ideales corroboraba, según él, que la hermandad hispanoamericana no era una nueva herramienta del imperialismo español, sino que era una necesidad sentida en todas las naciones hispanohablantes a fin de defenderse del materialismo anglosajón.

Vemos, por tanto, que la presencia de intelectuales hispanoamericanos en aquel Londres, y la facilidad con la que entraban en contacto los unos con los otros, estimulaba la formulación de proyectos que apelaban a una cultura compartida. También parece haber facilitado que definieran esa cultura compartida en contraposición con la del país en el que se encontraban, pese a que en la mayoría de casos los autores implicados también veían en Reino Unido numerosos aspectos de desarrollo técnico y económico que convenía admirar... y adoptar. Todo esto nos ayuda a entender no solo los artículos de Maeztu sobre las relaciones entre España y

40 Para esta revista, ver Rubiano Muñoz y Gómez García, 2016; Rausch, 2017 y 2018; Castaño Duque, 2014.

${ }^{41}$ Sobre esta figura, ver Jiménez Torres, 2016b. 
las repúblicas hispanoamericanas, sino también el hecho de que la pieza teatral que escribió durante estos años, El sindicato de las esmeraldas, estuviera protagonizada por unos patriotas de una ficticia república latinoamericana que viajan a Londres para obtener armamento con el que defenderse de la depredación de las multinacionales extranjeras. ${ }^{42}$ En una carta a Rojas, Maeztu expresó que con aquella obra esperaba que el público español se sintiera interpelado por un mensaje optimista y regeneracionista, por lo que podemos colegir que el autor partía de una coincidencia de particularidades, intereses y desafíos entre las naciones latinoamericanas representadas por sus personajes y el público español al que iba dirigida la obra - que nunca se llegó a representar.$-{ }^{43}$

\section{Debates británicos sobre los imperios}

Por importantes que resulten los contactos e influencias señalados hasta ahora, también conviene tener en cuenta que estos intelectuales no hablaban ni pensaban en un vacío. Porque no parece intranscendente que Maeztu y sus amigos latinoamericanos se encontraran, precisamente, en un país que se hallaba saturado de debates acerca del imperialismo y de propuestas acerca de cómo vertebrar las relaciones entre una serie de territorios unidos originalmente bajo un proyecto imperial, en un contexto marcado por nuevas amenazas geopolíticas. Desde los años 70 del siglo XIX se había ido afianzando la idea de que la expansión imperial había alcanzado sus límites, que la competencia entre imperios iba a ser cada vez más feroz, que la hegemonía británica mostraba síntomas de fragilidad y que varios competidores se preparaban para amenazarla, fuesen otras potencias europeas (Alemania, Francia) o nuevos actores (EE.UU., Rusia, Japón). También se fueron consolidando los movimientos nacionalistas en las distintas colonias británicas, y ganó peso la idea - derivada de los postulados positivistas de la época- de que era inevitable que con el andar del tiempo las colonias se terminaran gobernando a sí mismas, o que fueran los propios británicos quienes se hartasen del coste económico que suponía mantener el Imperio. Es curioso que, mientras en las presuntas «naciones latinas» se vivía

42 Maeztu, 2000, pp. 343-426. Análisis de esta obra, en González Cuevas, 2003, pp. 139-140, y Jiménez Torres, 2016a, pp. 62-64.

${ }^{43}$ Rojas, 1938, pp. 288-289. 
con angustia la pujanza imperial de Reino Unido (como presunto estandarte de la «raza anglosajona»), en la propia sociedad británica el futuro del Imperio era una fuente de enorme preocupación. ${ }^{44} \mathrm{Y}$ esto solo fue en aumento en los primeros años del siglo XX, sobre todo después de que la Guerra de los Boer (1899-1902) desencadenase toda una serie de ansiedades culturales en Reino Unido acerca de la capacidad militar e incluso racial de los propios británicos. Por otro lado, aquella guerra, unida a la tensión nacionalista en Irlanda, dio pie a fuertes debates acerca de la moralidad (o falta de ella) del imperialismo, y a reflexiones acerca del efecto que el nacionalismo imperialista (la palabra jingoísmo surgió precisamente en este contexto) estaba teniendo sobre la dirección política del país. La cuestión del pasado, el presente y el futuro del Imperio británico, por tanto, tenía muchas caras y afectó profundamente a todos los sectores ideológicos del país. ${ }^{45}$

La preocupación por el Imperio se tradujo en críticas y admoniciones, pero también en propuestas. A partir de la década de 1870 fueron en aumento las voces que planteaban reorganizaciones del Imperio en sentido federal o confederal, aunque con una gran diversidad en cuanto a cómo debía articularse la nueva relación entre Reino Unido y sus colonias. Autores como Charles Dilke o J. R. Seeley escribieron acerca de la necesaria articulación de una Greater Britain con base etnoracial - la de la raza inglesa o anglosajona que estaría esparcida por varios rincones del planeta.$-{ }^{46}$ Otros autores preferían hablar de sistemas de federación imperial o políticas de «preferencia imperial», como en la propuesta del ministro Joseph Chamberlain de reformar el sistema de aranceles, abandonando el tradicional librecambismo en favor de una política de comercio preferente entre Reino Unido y sus colonias. En cualquier caso, el eje que unía todas estas propuestas era la valoración de los lazos que unían el Imperio más allá de la imposición militar, y la apuesta por una mayor bilateralidad entre metrópolis y colonias que

44 Sobre la ansiedad latina ante la pujanza anglosajona, ver Litvak, 1980. Un ejemplo histórico y muy difundido en España, en Demolins, 1899.

45 Bell, 2009; Friedberg, 2010, pp. 89-134 y 209-78; Heyck, 2002, pp. 184-6. Sobre el periodo eduardiano como una era de grandes ansiedades nacionales, entre las que se hallaba la preocupación por el Imperio, ver Brooks, 1995, y Powell, 1996.

${ }^{46}$ Las ambigüedades del término race, utilizado en este contexto, no eran muy distintas de las que atravesaban el uso de raza en el contexto español; ver Bell, 2009, pp. 134-40. 
implicase, también, la corresponsabilidad de estas en su propia gobernanza. El objetivo era, por tanto, redefinir la relación entre Reino Unido y su Imperio: del mandato y la imposición debía pasarse a la alianza política y la colaboración económica.

Ninguna de estas propuestas tuvo éxito, o al menos no en las últimas décadas del siglo XIX o las primeras del XX. Por lo tanto, si bien hoy sabemos que Reino Unido estaba sentando durante aquellos años las bases para la exitosa transformación de su Imperio en la actual Commonwealth (el Estatuto de Westminster, pieza clave en la transformación de los dominions en naciones soberanas, se terminaría aprobando en 1931), es natural que un observador presente en aquel país durante aquellos años recibiese la impresión de que la gran potencia imperial no sabía qué hacer con sus colonias, ni cómo asegurar su futuro. Los propios defensores de una Greater Britain o de esquemas federalistas se mostraron repetidamente incapaces de lograr cambios sustanciales en la política exterior o la realidad legislativa del Imperio, y fueron vistos por sus contemporáneos generalmente como políticos y pensadores fracasados.$^{47}$ No sorprende, por tanto, que Maeztu escribiese en 1911 sobre la cuestión imperial como «el problema que hace bambolearse la civilización sajona», y que en Defensa de la Hispanidad también hiciese referencia a «Inglaterra, con su Imperio resquebrajado». ${ }^{48}$ Como en el caso del hispanoamericanismo durante la misma época, hubo una notable tensión entre un discurso de gran arraigo y transversalidad en la esfera pública y una escasez de consecuencias tangibles. ${ }^{49}$

También es relevante que aquellos pensadores y políticos británicos que más criticaban el modelo imperial estaban atravesados de una serie de tensiones muy características. Como ha afirmado Porter, para la mayoría de británicos de la época «la verdadera alternativa al «imperialismo» no era el «antiimperialismo», sino que tenía que ser algo distinto». ${ }^{50}$ Después de todo, la expansión colonial europea durante el siglo XIX había demostrado que el mundo estaba lleno de naciones soberanas o de empresas internacionales dispuestas a abalanzarse sobre cualquier territorio que estu-

47 Bell, 2009, p. 27.

48 Maeztu, 2005, p. 134.

49 Sobre la brecha entre la retórica grandilocuente y exitosa del hispanoamericanismo y la pobreza de los avances concretos, ver Moreno Luzón, 2010, pp. 627-32.

50 Porter, 2008, p. xxx. En el original, «the true alternative to "imperialism" was not "anti-imperialism", but had to be something else». Traducción mía. 
viese disponible, o incluso que fuese medianamente vulnerable (como se había visto, precisamente, en la Guerra de 1898). Esta visión subrayaba que un antiimperialismo total sería poco menos que irresponsable, dado que la dominación imperial era un hecho de las relaciones internacionales. Lo único que podía variar era qué naciones, grupos raciales o empresas dominaban qué lugares. Según Porter, para los eduardianos era evidente que «el imperialismo no se detenía solo porque algún imperio lo hiciese». ${ }^{51}$ Además, las teorías positivistas y darwinianas acerca de la imparable marcha de la modernidad descartaban automáticamente que los nativos de tierras colonizadas por europeos pudieran no salir beneficiados de aquel contacto.

La cuestión, pues, era cómo se podía dar una nueva forma al imperialismo de manera que fuese tan ventajoso para el colonizado como para el colonizador. En esta línea, George Bernard Shaw propuso en su ensayo Fabianism and the Empire (1900) la reconversión del imperio británico en un imperio socialista, en el sentido fabiano del socialismo como un mecanismo de gestión social eficiente y humanitario. La idea era fomentar una alianza de las distintas razas que conformaban el Imperio, de la cual saldría una nueva burocracia que garantizase las necesidades básicas de todos los ciudadanos imperiales. No muy distinta fue la postura de J. A. Hobson en su Estudio del imperialismo (1902); si bien criticaba duramente las motivaciones capitalistas del imperialismo europeo - sus tesis influirían decisivamente en las de Lenin-, también se oponía a que los Estados se retiraran de sus colonias, puesto que esto dejaría el camino libre a empresas internacionales para que explotaran a aquellas sin control alguno. Además, Hobson sostuvo que la intervención de las potencias europeas estaba justificada en caso de que una nación «incivilizada» no estuviese poniendo sus recursos al servicio de la humanidad. Para él, el imperialismo era aceptable mientras contribuyese a la «protección, educación y autodesarrollo» de las «razas inferiores».

Maeztu prestó mucha atención a todos estos debates durante su estancia británica. El primer asunto que tuvo que cubrir como corresponsal en Londres fue, precisamente, la propuesta de reforma arancelaria en un sentido federalizante de Chamberlain, que suponía una amenaza

51 Porter, ob. cit., p. xxxvi. En el original, «imperialism' did not stop just because empires seemed to». Traducción mía. 
para los intereses comerciales españoles. Además, algunas de las figuras que más llamaron su atención durante su estancia británica fueron precisamente aquellos que criticaban la configuración del Imperio según las líneas ya descritas: Shaw, Hobson, Sidney Webb, Cunninghame Graham, los socialistas Ramsay MacDonald y Keir Hardie, Lord Rosebery e incluso los ya mencionados Chesterton y Belloc, quienes se mostraron muy críticos con la Guerra de los Boer y la política imperial en Sudáfrica. ${ }^{52}$ Esto nos permite entender por qué la admiración que Maeztu expresó por la sociedad y la política británicas durante buena parte de su etapa inglesa se centrara casi siempre en cuestiones no relacionadas con el Imperio (por ejemplo, el ímpetu de sus reformistas sociales o la independencia e intensidad de su esfera pública); y también nos anima a considerar las coincidencias entre las ideas de estos autores y las que Maeztu desarrollaría acerca del antiguo imperio español. Porque los eduardianos críticos con el imperialismo de su propio país también creían el Imperio británico no se preocupaba por realizar una verdadera misión civilizadora, sino que más bien estaba alentado por motivaciones materialistas. En un texto escrito en el contexto de la Guerra de los Boer, por ejemplo, Chesterton argumentó que los imperialistas británicos «entienden por el amor a su país, no algo semejante al amor que siente un místico por Dios, sino más bien el amor que siente un niño por la mermelada». ${ }^{53}$ Además, tanto Chesterton como los otros autores críticos con el imperialismo británico argumentaban que la separación racial que había producido esta mentalidad amenazaba la viabilidad del Imperio a largo plazo. Cuando Maeztu describía esta separación como «el problema que hace bambolearse la civilización sajona», por tanto, estaba expresando un diagnóstico compartido por muchos británicos del momento. Y tanto en ellos como en Maeztu encontramos una cierta ambivalencia con respecto a los mecanismos y a la historia del imperialismo europeo: el imperialismo no se debía rechazar como una gigantesca aberración histórica, sino que debía ser sustituido por una alianza mutuamente beneficiosa entre aquellos pueblos que habían sido unidos inicialmente por un proyecto imperialista.

52 Lothian, 2009, p. 19. Para los artículos de Maeztu acerca de estas figuras, ver Jiménez Torres 2016a, pp. 47-86.

${ }_{53}$ Chesterton, 2012. En el original, «that they mean by the love of country, not what a mystic might mean by the love of God, but something of what a child might mean by the love of jam». Traducción mía. 
Todo esto sugiere que hubo cierta permeabilidad entre los debates acerca del Imperio británico que se estaban produciendo a comienzos del siglo Xx y la dirección que tomaría el pensamiento de Maeztu a la hora de concebir las relaciones entre España y sus antiguas colonias. Y, desde luego, ayuda a entender que alguien que pasó tantos años en Reino Unido y estuvo tan vinculado a corrientes británicas de pensamiento escribiese en Defensa de la Hispanidad que existía un «secular conflicto entre la Hispanidad y los pueblos anglosajones». ${ }^{54}$ Maeztu no se estaba refiriendo a la enemistad histórica entre España y Gran Bretaña, ni a la relación entre Estados Unidos y las repúblicas latinoamericanas; en su visión, estas eran meras expresiones históricas de un enfrentamiento de cariz ontológico entre filosofías enfrentadas y visiones diametralmente opuestas de la vida. Pero esta postura no se producía como rechazo del discurso imperante en aquel momento en el mundo británico, sino que más bien se veía influido, o al menos apoyado, por él. A la hora de construir su visión del Imperio británico como modelo negativo para las naciones hispanoamericanas, y de la deseabilidad de que territorios unidas originalmente por un proyecto imperial se uniesen en una cooperación entre naciones soberanas, Maeztu estaba recogiendo muchos elementos del debate público británico de comienzos de siglo.

\section{Conclusiones}

Los artículos de Maeztu publicados entre 1911 y 1912 nos animan a reconsiderar la génesis de su idea de la Hispanidad y a plantearla sobre unos ejes distintos de los que se han solido señalar. Maeztu no habría desarrollado sus ideas acerca de las relaciones entre España y las repúblicas hispanoamericanas a finales de los años 20 , en el marco del proyecto de la derecha monárquica durante la dictadura primorriverista, sino que buena parte de esas ideas se desarrollaron entre 1905 (fecha de su llegada a Londres) y 1912. Que Maeztu se interesase por las ideas hispanoamericanistas durante su estancia en Londres puede deberse, en parte, al contexto de los centenarios de la independencia de las repúblicas hispanoamericanas; pero hay motivos para pensar que esta no fue la única razón ni influen-

${ }^{54}$ Maeztu, 2005, p. 126. 
cia sobre su pensamiento. También fue decisivo su contacto con escritores y políticos latinoamericanos como Rojas, Arguedas, García Calderón y el resto de responsables de la revista Hispania, residentes todos en Londres: estos le expusieron a las principales coordenadas de los proyectos hispanoamericanistas, y reforzaron la idea de que reivindicar una mayor cooperación entre España y sus antiguas colonias no suponía un regreso a un proyecto imperial, sino que se trataba de algo deseado también al otro lado del Atlántico. Además, la presencia de Maeztu en Londres le expuso a los debates británicos acerca del pasado, presente y futuro de su propio Imperio, incluyendo las críticas y propuestas alternativas que se llevaban formulando desde finales del siglo XIX. Todo esto facilitó - bien que de forma paradójica y contraintuitiva - que Maeztu aceptase una idea de la identidad anglosajona y de las características históricas del Imperio británico como radicalmente opuestas a la identidad de los países de habla hispana y el antiguo Imperio español.

Esto nos anima a insertar las tesis que acabaron confluyendo en la idea de la Hispanidad en un marco más amplio que el de la historia intelectual española o, incluso, hispanoamericana. Más bien podemos verlas como parte de una corriente europea de comienzos del siglo XX que buscaba, desde experiencias nacionales muy distintas, repensar el pasado, presente y futuro de la colonización europea. Esta corriente se mostraba crítica con los proyectos imperiales del pasado pero, al mismo tiempo, abogaba por utilizarlos como una base desde la cual crear una nueva serie de alianzas internacionales, y los resignificaba dentro de los imaginarios nacionales (y nacionalistas) de las antiguas metrópolis, facilitando su tránsito hacia las identidades posimperiales de la segunda mitad del siglo Xx. Esto nos anima también a recalibrar las coordenadas del pensamiento hispanoamericanista, y ponderar las influencias sobre el mismo de otras realidades nacionales y culturales más allá de España y las repúblicas latinoamericanas, sobre todo en los casos de aquellos autores que tuvieron una importante exposición a otras culturas europeas.

\section{Fuentes}

Epistolario Maeztu-Ortega y Gasset, Fundación Ortega y Gasset-Gregorio Marañón.

Hemeroteca Digital, Biblioteca Nacional de España. 


\section{Bibliografía}

ABELLÁN, José Luis, Sociología del 98, Barcelona, Península, 1973.

ARGUEDAS, Alcides, Pueblo enfermo:contribución a la psicología de los pueblos hispano-americanos, Barcelona, Luis Tasso, 1909.

BELL, Duncan, The Idea of Greater Britain: Empire and the Future of World Order, 1860-1900, Princeton, Princeton University Press, 2009.

BERMEJO MARÍN, Pedro, «Londres, capital originaria del hispanoamericanismo», Cuadernos hispanoamericanos, 805-806, 2017, pp. 84-97.

BROOKS, David, The Age of Upheaval: Edwardian Politics, 1899-1914, Mánchester, Manchester University Press, 1995.

BRUNO, Paula, «Un momento latinoamericano. Voces intelectuales entre la I Conferencia Panamericana y la Gran Guerra», en FUENTES, M. y ARCHILÉS, F. (eds.), Ideas comprometidas. Los intelectuales y la política, Madrid, Akal, 2018, pp. 57-77.

CASTAÑO DUQUE, Gildardo, «Revista Hispania (1912-1916): presencia cultural colombiana en la vida intelectual europea», en VIVAS HURTADO, Selnich (coord.), Utopías móviles: nuevos caminos para la Historia intelectual en América Latina, Medellín, Diente de León/Universidad de Antioquia, 2014, pp. 32-63.

CASTRO MONTERO, Ángeles (coord.), Españoles en el diario La Prensa, Buenos Aires, Fundación Ortega y Gasset Argentina, 2012.

CHESTERTON, Gilbert Keith, «A defence of patriotism», en The Defendant, Mineola, N.Y.: Dover Publications, 2012, pp. 78-82.

CORRIN, Jay P., G.K. Chesterton and Hilaire Belloc: The Battle Against Modernity, Londres, Ohio University Press, 1981.

CORRIN, Jay P., Catholic Intellectuals and the Challenge of Democracy, Notre Dame, University of Notre Dame Press, 2002.

DEMOLINS, Edmond, En qué consiste la superioridad de los anglosajones, Valladolid, Impresora Castellana, 1899.

FERGUSON, Robert, The Short, Sharp Life of T.E. Hulme, Londres, Allen Lane, 2002.

FRAGA, Manuel, Ramiro de Maeztu y el pensamiento político británico, Vitoria, Consejo de Cultura de la Diputación Foral de Álava, 1974.

FRAGA, Manuel, Ramiro de Maeztu en Londres, Madrid, Cultura Hispánica, 1976.

FREEDEN, Michael, The New Liberalism: an Ideology of Social Reform, Oxford, Clarendon Press, 1978.

FRIEDBERG, Aaron, The Weary Titan: Britain and the Experience of Relative Decline, Princeton, Princeton University Press, 2010

GARCÍA CALDERÓN, Francisco, Profesores de idealismo, París, Sociedad de Ediciones Literarias y Artísticas, 1909. 
GONZÁLEZ CALLEJA, Eduardo y LIMÓN NEVADO, Fredes, La Hispanidad como instrumento de combate, Madrid, CSIC, 1988.

GONZÁLEZ CUEVAS, Pedro Carlos, Maeztu. Biografía de un nacionalista español, Madrid, Marcial Pons, 2003.

HEYCK, Thomas William, A History of the peoples of the British Isles, vol. 3, London, Routledge, 2002.

JIMÉNEZ TORRES, David, Ramiro de Maeztu and England. Imaginaries, Realities and Repercussions of a Cultural Encounter, Woodbridge, Tamesis, 2016a.

JIMÉNEZ TORRES, David, «De historiador de la conquista a icono de la Hispanidad: R. B. Cunninghame Graham como mediador cultural». Erebea: Revista de Humanidades y Ciencias Sociales 6, 2016 b.

LANDEIRA, Ricardo, Ramiro de Maeztu, Boston, Twayne, 1978.

LITVAK, Lily, Latinos y anglosajones: orígenes de una polémica, Barcelona, Puvill, 1980.

LOMBARDERO ÁLVAREZ, Jorge, «Maeztu y la Hispanidad», El Basilisco, vol. 2, núm. 25, 1999, págs. 51-60.

LOTHIAN, James, The Making and Unmaking of the English Catholic Intellectual Community (1910-1950), Notre Dame, University of Notre Dame Press, 2009.

MADARIAGA, Salvador de, Españoles de mi tiempo, Barcelona, Planeta, 1974.

MAEZTU, Ramiro de, Inglaterra en armas, Londres, Darling \& Son, 1916.

MAEZTU, Ramiro de, Obra literaria olvidada, Madrid, Biblioteca Nueva, 2000.

MAEZTU, Ramiro de, Defensa de la Hispanidad, Madrid, Homo Legens, 2005.

MAEZTU, Ramiro de, Hacia otra España, Madrid, Biblioteca Nueva, 2007.

MAINER, José-Carlos, «El vuelo epicúreo de Ramiro de Maeztu», en R. de Maeztu, Don Quijote, Don Juan y la Celestina: ensayos en simpatía, Madrid, Comunidad de Madrid - Visor Libros, 2004.

MARCILHACY, David, Raza hispana: hispanoamericanismo e imaginario nacional en la España de la Restauración, Madrid, Centro de Estudios Políticos y Constitucionales, 2010.

MARCILHACY, David, «Las figuras de la "raza": de la España Mayor a la Comunidad Iberoamericana, perspectivas (post)imperiales en el imaginario español», Historia y Política 35, 2016, pp. 145-174.

MARTIN, Wallace, The New Age under Orage: Chapters in English Cultural History, Mánchester, Manchester University Press, 1967.

MORENO LUZÓN, Javier, «Reconquistar América para regenerar España. Nacionalismo español y centenarios de las independencias en 1910-1911», Historia Mexicana, vol. LX, no. 1, 2010, pp. 561-640.

NIÑO RODRÍGUEZ, Antonio, 1993 «Hispanoamericanismo, regeneración y defensa del prestigio nacional (1898-1931)», en PÉREZ HERRERO, P., y TABANERA, N. (coords), España/América Latina: un siglo de políticas culturales, Madrid: Aieti-Síntesis, pp. 15-48. 
NYE. Joseph, Soft Power: The Means to Success in World Politics, New York, Public Affairs, 2004.

OCIO, Luis, Ramiro de Maeztu. Un monárquico en la II República, Bilbao, Servicio Editorial de la Universidad del País Vasco, 2014.

OLARIAGA, Luis, «Cómo era y cómo pensaba Ramiro de Maeztu en su etapa en Inglaterra», en VAL, V. (coord.) En torno a Ramiro de Maeztu, Vitoria, Institución Sancho el Sabio, 1974, pp. 47-61.

PIKE, Frederick, Hispanismo, 1898-1936: Spanish Conservatives and Liberals in their Relations with Spanish America, Notre Dame, University of Notre Dame, 1971.

PLA, José, «Florilegio epistolar de Ramiro de Maeztu». Cuadernos Hispanoamericanos 33-34, 1952, pp. 55-76.

PORTER, Bernard, Critics of Empire: British Radicals and the Imperial Challenge, Londres, I. B. Tauris, 2008.

POWELL, David, The Edwardian Crisis: Britain, 1901-1914, Basingstoke, Macmillan, 1996.

RAUSCH, Jane M., «An Overlooked Contributor to a Unique Colombian Periodical. Enrique Pérez Lleras and the journal Hispania (1912-1916)». Historia Crítica 68, 2018, pp. 95-110.

RAUSCH, Jane M., Santiago Pérez Triana (1858-1916): Colombian Man of Letters and Crusader for Hemispheric Unity, Princeton, Markus Wiener Publishers, 2017.

ROJAS, Ricardo, Retablo español, Buenos Aires, Losada, 1938.

RUBIANO MUÑOZ, Rafael y GÓMEZ GARCÍA, Juan Guillermo, Años de vértigo: Baldomero Sanín Cano y la revista Hispania (1912-1916), Bogotá, Siglo del Hombre Editores, 2016.

SEARLE, Geoffrey Russell, The Quest for National Efficiency, Oxford, Blackwell, 1971.

SEPÚLVEDA, I., El sueño de la madre patria: hispanoamericanismo y nacionalismo, Madrid, Fundación Carolina - Centro de Estudios Hispánicos e Iberoamericanos, 2005.

SIERRA GONZÁLEZ, Elisa, El impacto del mundo anglosajón en la obra de Ramiro de Maeztu, Tesis doctoral inédita, City University of New York, 1984.

VEGAS LATAPIE, Eugenio, Memorias políticas: el suicidio de la monarquía y la Segunda República, Barcelona, Planeta, 1983. 


\section{Financiación}

Este artículo forma parte de los resultados del proyecto «La patria hispana, la raza latina. Intelectuales, identidades colectivas y proyectos políticos entre España, Italia y Argentina (1880-1945)» (Programa estatal de fomento de la investigación científica y técnica de excelencia - Subprograma estatal de generación de conocimiento. Ministerio de Industria, Economía y Competitividad, ref. HAR2016-75324-P).

\section{Datos del autor}

David Jiménez Torres. Investigador Juan de la Cierva en la Universidad Complutense de Madrid. Doctor en Estudios Hispánicos por la University of Cambridge. Ha sido profesor en University of Cambridge, University of Manchester y Universidad Camilo José Cela. Autor de Ramiro de Maeztu and England: Imaginaries, Realities and Repercussions of a Cultural Encounter (Boydell \& Brewer, 2016), coordinador junto a Leticia Villamediana de The Configuration of the Spanish Public Sphere: from the Enlightenment to the Indignados (Berghahn Books, 2019) y coordinador junto a Marta Pérez-Carbonell del dossier «Representing Violence in Twenty-First-Century Spain: Novels, Films and Performance Arts" en Bulletin of Spanish Studies $(96: 7,2019)$. Autor también de numerosos capítulos de libro y artículos científicos en revistas como Historia y Política, Revista de Hispanismo Filosófico, Hispanic Research Journal o Bulletin of Spanish Studies. 